\title{
Radiation Dose-Volume Effects for Liver SBRT
}

\author{
Moyed Miften, $\mathrm{PhD}^{\star}$, Yevgeniy Vinogradskiy, $\mathrm{PhD}^{\star}$, Vitali Moiseenko, $\mathrm{PhD}^{\dagger}$, Jimm Grimm, \\ $\mathrm{PhD}^{\ddagger}$, Ellen Yorke, PhD§, Andrew Jackson, PhD§, Wolfgang A. Tomé, PhD\|, Randall K. Ten \\ Haken, PhDI, Nitin Ohri, MD\|, Alejandra Méndez Romero, MD\#, Karyn A. Goodman, MD*, \\ Lawrence B. Marks, MD**, Brian Kavanagh, MD*, Laura A. Dawson, MD ${ }^{\dagger \dagger}$
}

*Department of Radiation Oncology, University of Colorado Denver, Aurora, Colorado tDepartment of Radiation Medicine and Applied Sciences, University of California, San Diego, La Jolla, California $\neq$ Department of Radiation Oncology and Molecular Radiology Sciences, Baltimore, Maryland §Department of Radiation Oncology, Memorial Sloan Kettering Cancer Center, New York, New York "Department of Radiation Oncology, Montefiore Medical Center and Albert Einstein College of Medicine, Bronx, New York IDepartment of Radiation Oncology, University of Michigan, Ann Arbor, Michigan \#Department of Radiation Oncology, Erasmus Medical Center Cancer Institute, Rotterdam, The Netherlands " Department of Radiation Oncology, University of North Carolina, Chapel Hill, North Carolina t'Radiation Medicine Program, Princess Margaret Cancer Centre and Department of Radiation Oncology, University of Toronto, Toronto, Ontario, Canada

\begin{abstract}
Stereotactic body radiation therapy (SBRT) has emerged as an effective, noninvasive treatment option for primary liver cancer and metastatic disease occurring in the liver. Although SBRT can be highly effective for establishing local control in hepatic malignancies, a tradeoff exists between tumor control and normal tissue complications. The objective of the present study was to review the normal tissue dose-volume effects for SBRT-induced liver and gastrointestinal toxicities and derive normal tissue complication probability models.
\end{abstract}

\section{Clinical Significance}

Each year, an estimated 696,000 deaths from primary liver cancer are recorded in the world, making it the third most common cause of cancer death worldwide (1). Moreover, the liver is second only to regional lymph nodes as a site of metastatic disease (2), often from primary cancer of the bowel, breast, lung, kidney, and skin (melanoma). Aggressive treatment of primary and metastatic liver lesions is associated with durable disease-free survival in selected patients with typically small liver lesions $(<5 \mathrm{~cm})$ and no or minimal extrahepatic disease $(3,4)$. Although surgery remains an effective treatment option for some patients (5), SBRT has emerged as an effective, noninvasive alternative for these tumors (6-15). In SBRT,

Reprint requests to: Moyed Miften, PhD, Department of Radiation Oncology, University of Colorado Denver School of Medicine, Aurora, CO 80045., Moyed.Miften@ucdenver.edu.

Conflict of interest: none. 
a high dose is delivered in 5 fractions using sophisticated dose delivery systems that yield steep dose gradients near the tumor edge, thus limiting the dose to the surrounding organs at risk (OARs) (16).

SBRT can be highly effective in providing local control in selected patients with small hepatic malignancies. A dose-response effect exists, with increasing doses yielding greater rates of local control (17). However, SBRT also carries risks to the adjacent normal tissues, and when selecting doses for treatment, there are tradeoffs between tumor control and normal tissue complications $(18,19)$. A variety of prescription doses and fractionation schemes with a wide range of toxicity endpoints have been reported in the published data. The objective of the present study was to review the normal tissue dose-volume effects from liver SBRT and derive normal tissue complication probability models.

\section{Endpoints}

Numerous toxicities can occur from radiation therapy to the liver. However, sufficient data were available to analyze 2 toxicities: liver enzyme changes and gastrointestinal (GI) toxicity. A variety of endpoints have been used in studies, including progression of the Child-Pugh score (12-14, 20, 21), classic and nonclassic radiation-induced liver disease (RILD) $(11,19,22)$, liver enzyme changes (defined as elevation in alanine aminotransaminase or aspartate aminotransaminase) (11-14, 20, 21, 23), and/or Common Terminology Criteria for Adverse Events (CTCAE) grade progression for hepatic, thrombocytopenic, or general GI toxicity. General GI toxicities included fatigue, nausea, diarrhea, gastritis, ulcers, GI area pain, and colitis (9-12, 14, 21-26). The range in the type of toxicities reported made it challenging to aggregate the data meaningfully. At the writing of our report, the 3 endpoints most frequently cited and that we chose to use for analysis were grade $\geq 3$ liver enzyme elevation, grade $\geq 2$ general GI toxicities, and grade $\geq 3$ general GI toxicities. Grade $\geq 3$ liver enzyme hepatic toxicity has been defined as $\geq 5$ times the upper limit of the normal level for alanine aminotransferase and aspartate aminotransferase according to the CTCAE, version 4.0. The general GI toxicity endpoint included fatigue, nausea, GI area pain, diarrhea, gastric ulcer, and colitis.

\section{Challenges Defining Volumes}

Variability in image segmentation can affect the dose-volume/outcome complication relationships for the liver and other upper abdominal normal tissues (esophagus, stomach, duodenum, and small intestine). The use of breath hold computed tomography (CT) imaging and oral and intravenous contrast are recommended to aid in reproducible and accurate segmentation of the upper abdominal normal tissues, some of which are irregularly shaped and difficult to define on cross-sectional images. Suggested guidelines for normal tissue segmentation were described by Jabbour et al (27). Target motion should be accounted for in the generation of an internal target volume, and the capability of the tracking, gating, and/or breath hold techniques and any residual uncertainties should be taken into account when generating the planning target volume (PTV). Critical structure motion should be considered by adding a margin to form a planning OAR volume. 
In terms of the liver toxicity assessment, the liver is usually large relative to the tumor and relative to the moderate-to-high dose portions of the 3-dimensional (3D) dose distribution in the setting of liver metastases. In contrast, the liver is often cirrhotic and smaller in patients with primary hepatobiliary cancer. Thus, modest inaccuracies or inconsistencies in the segmentation of liver could be more likely to have clinically relevant effects on the dosevolume metrics in patients with cirrhosis than in patients with metastases. Nonetheless, patients with metastases often have undergone previous surgery and, consequently, will have a smaller and unusually shaped liver. Reporting the absolute volume of liver receiving varying radiation doses has been recommended (19). It has also been recommended that the gross tumor volume (GTV) should be excluded from the liver volume, because the GTV is generally nonfunctional. In addition, much of the original dose-volume and normal tissue complication probability liver research was performed using the liver minus the GTV. Thus, including the GTV in the liver contour will overstate the exposure to the normal liver.

To the degree that toxicity to other organs in the upper abdomen can cause symptoms that could be attributed to liver injury (or tumor progression), care should be taken to monitor the doses to these other organs (eg, stomach, duodenum, bowel, biliary tree). Because these structures can be challenging to define on $\mathrm{CT}$ images, with much greater interobserver variation in the total volume, the dose metrics based on the absolute volume should be assessed, in addition to the relative dose-volume parameters.

\section{Review of Outcomes Data}

Potential studies to include in the present review were identified by performing a keyword search for "SBRT and liver" and "stereotactic ablative body radiotherapy (SABR) and liver" in PubMed up to May 2014. Only studies that contained both toxicity and dose-volume data were included (Table 1).

The typical treatment techniques are described and summarized in Table 2. Simulation CT imaging usually included some form of motion management (eg, 4-dimensional CT imaging, abdominal compression, or an active breathing control system). In general, prescription doses were prescribed to cover the PTV with the $70 \%$ to $85 \%$ isodose line (11, 20). Treatment delivery was performed using linear accelerator-based systems or specialized SBRT delivery systems with image guidance at each fraction using either orthogonal planar imaging or 3D cone beam CT imaging. In certain cases, implanted fiducial markers were used as a guide for real-time target tracking $(10,21,22)$.

We found only 2 groups in our search that had directly included dose-response modeling in their studies, Son et al (20) and the Princess Margaret Cancer Centre group (11-13). Son et al (20) analyzed the association between the dose-volume parameters and liver toxicity within 6 months after SBRT in 36 patients with hepatocellular carcinoma (HCC) receiving SBRT to doses of 30 to $39 \mathrm{~Gy}$ in 3 fractions. They analyzed grade $\geq 2$ hepatic toxicity (CTCAE, version 3.0) or progression beyond pretreatment toxicity for patients with baseline grade 2 and Child-Pugh class progression as a function of the total liver volume receiving less than a certain dose $\left(\mathrm{rV}_{\text {dose }}\right) \cdot \mathrm{rV}_{\text {dose }}$ is a metric that is the reverse of the standard percentage volume receiving at least a certain dose $\left(\mathrm{eg}, \mathrm{V}_{20 \mathrm{~Gy}}\right)$ concept. Multivariate logistic 
regression suggested that the absolute liver volume receiving $<10 \mathrm{~Gy}\left(\mathrm{rV}_{10 \mathrm{~Gy}}\right)$ was predictive of grade $\geq 2$ hepatic toxicity $(P=.022)$ and $\mathrm{rV}_{18 \mathrm{~Gy}}$ was predictive of Child-Pugh class progression $(P=.05)(20)$. Son et al (20) also presented sigmoidal dose-response curves to model grade $\geq 2$ toxicity and Child-Pugh progression as a function of $\mathrm{rV}_{\mathrm{dose}}$. Because no other investigators presented their analysis in terms of $\mathrm{rV}_{10 \mathrm{~Gy}}$ and $\mathrm{rV}_{18 \mathrm{~Gy}}$, we could not directly include these results in our analysis. However, we did include the mean liver dose (MLD) results from Son et al (20) in our model. All studies excluded the GTV in the MLD calculation, with the exception of the study by Son et al (20).

The Princess Margaret Cancer Centre group (11-13) reported data from a phase I doseescalation study using the toxicity estimates from previously derived models. They used the Lyman-Kutcher-Burman formulation to model the probability of RILD as a function of the effective liver volume irradiated $\left(\mathrm{V}_{\text {eff }}\right)$ using modeling parameters from the University of Michigan experience (28) and correcting for fractionation using an $\alpha / \beta$ ratio of $2.5 \mathrm{~Gy}$. The patients were then divided into 1 of 3 strata according to their $\mathrm{V}_{\text {eff }}$ (low-, mid-, and highvolume irradiation) and total doses (in a constant of 6 fractions) were escalated within each strata corresponding approximately to an iso-toxicity rate of 5\%, 10\%, or $20 \%$ (11). Using the dose-escalation scheme described, no classic RILD was observed, indicating that the model overestimated the risk of classic RILD. However, nonclassic RILD did occur (11). The investigators reported an overall grade $\geq 3$ toxicity rate (including biochemical, hematologic, and general GI toxicity) of 30\% for their HCC cohort (12) and 3\% grade 3 liver enzyme changes for their metastasis cohort (11). No direct comparison of their model results to ours was possible, because no other study provided outcomes as a function of $\mathrm{V}_{\text {eff }}$. However, the enzyme toxicity outcomes from the Princess Margaret Cancer Centre group were included in our modeling.

A decline in liver function (eg, worsening Child-Pugh score) after SBRT has been reported in $10 \%$ to $30 \%$ of patients with primary liver cancer $(12,14,20,24)$. Grade 5 liver toxicity due to liver failure has also been observed less commonly after SBRT in patients with primary liver cancer. We have summarized, in brief, the 3 studies that explicitly reported grade 5 liver failure $(12,14,24)$. Bujold et al (12) reported 3 deaths of 102 patients with $\mathrm{HCC}$ as a result of liver failure (using Child-Pugh scoring) with no noted cancer progression. Barney et al (24) reported 1 case of grade 5 liver failure in 9 patients. Andolino et al (14) reported liver failure in 4 of $8 \mathrm{HCC}$ patients with a baseline Child-Pugh score of $\geq 8$, with 2 of these patients dying of liver cancer and 2 undergoing liver transplantation. No specific common cause for liver failure was identified in the 3 reports, although some were associated with a worse baseline Child-Pugh score, tumor vascular thrombosis progression, and extensive previous systemic therapy. Liver failure was not explicitly included in the dose-response modeling because there was not enough consistently reported data. More quantitative studies have been reported highlighting the relationship between baseline liver function and liver toxicity in patients with primary liver cancer since our analysis (29-31).

\section{Factors Affecting Outcomes}

Several factors, other than the dose and volume, have been suggested to be predictors of toxicity (29). Several studies noted that pretreatment Child-Pugh status and pre-existing GI 
conditions were risk factors for the subsequent development of post-treatment hepatic and nonhepatic GI toxicity $(10,12,14,21,29,30)$. There is a general agreement that patients with primary liver tumors are more likely to develop RT-associated liver injury than are patients with metastatic lesions (presumably because of underlying liver disease and smaller volume of healthy liver to spare in the former group) $(19,32)$. Other clinical and patient factors noted to possibly increase the risk of toxicity were increasing tumor size (25), tumor location within the liver (patients with central tumors near the liver hilum had a greater risk of GI toxicity [10]), and pretreatment systemic therapies (24).

\section{Mathematical/Biologic Model}

Based on the available data, 3 pooled dose-response relationships were analyzed: (1) grade 33 liver enzyme toxicity as a function of physical MLD (Fig. 1); (2) grade $\geq 2$ general GI toxicity as a function of the prescription (Rx) dose (Fig. 2); and (3) grade $\geq 3$ general GI toxicity as a function of the Rx (Fig. 3). All studies used in the grade $>3$ liver enzyme analysis reported MLD using uninvolved liver, with the exception of Son et al (20). For the GI toxicity analysis, the Rx doses reported in the studies were used because the doses to specific OARs were not available in many cases. Because the Rx isodoses were relatively similar across the studies and the lesion location could also have been relatively similar across the studies, a reasonable correlation might exist between the prescribed target doses and the doses to the normal liver. Physical doses were used for modeling rather than biologically effective doses (BEDs) owing to the uncertainty of the $\alpha / \beta$ ratio for the organs (eg, esophagus, stomach, duodenum, small intestine) responsible for GI toxicity and because conversion of the mean dose to the BED was not possible owing to the lack of full 3D dose data. The number of fractions for the included studies was concentrated around 3 to 6 fractions, with the exception of the study by Goodman et al (10), which used single-fraction treatments (Table 2). Several reports provided overlapping study sets. For each overlapping study set, we used the most recent report with the available toxicity endpoint and excluded the older studies with overlapping data.

Dose-response modeling was performed using a probit model with maximum likelihood parameter fitting (33-35). The maximum likelihood method determined the probit model parameters that best fit the binomial data (toxicity vs no toxicity). The data input into the model were the reported toxicity rates and corresponding dose metrics reported in each included study. The average toxicity rate was binned into binary outcomes to facilitate probit model estimation with maximum likelihood parameter fitting. The confidence intervals (CIs) for the model fitting parameters were calculated using the profile likelihood method. The dose "error bars" (horizontal axis) for each data point in the presented graphs represent the range of reported doses for each study, and the toxicity "error bars" (vertical axis) represent the binomial 68\% CIs. The CIs of the dose-response curves were calculated using 2000 samples of the bootstrap method, with random sampling with replacement, for each toxicity type. All model fit parameters are listed in Table 3.

Five studies were included in the grade 23 liver enzyme analysis (Fig. 1) $(10-12,14,20)$. The data points visually suggested an increasing probability of grade $\geq 3$ liver enzyme toxicity as a function of MLD. However, the probit model fitting did not produce a 
statistically significant dose-response fit, indicating that the model failed to establish the upper confidence limit for the dose resulting in 50\% probability of complication $\left(\mathrm{D}_{50}\right)$ or improve on the model assuming no dose dependence with statistical significance at $P=.10$. Therefore, we could not exclude that the incidence of liver enzyme complications was independent of the dose. We have reported the probit model fit for reference, with the $68 \%$ and $95 \%$ CIs for the model. Possible explanations for the insignificant fit included the sparsity of data (the analysis included only 5 studies), the low incidence of complications, and variations in baseline liver function and the definitions used for liver enzyme abnormalities. Furthermore, it is possible that the model fit was skewed by the outlier data point, which represents the study by Lee et al (11). As expected, the 2 studies that included metastatic patients $(10,11)$ reported lower toxicity rates than those reported by the studies that included primary tumor patients.

Seven studies were included in the analysis relating grade $\geq 2$ general GI toxicity to the $\mathrm{Rx}$ dose $(10,11,13,14,22,24,25)$. A statistically significant probit model fit was found (Fig. 2). The model fitting parameters were $\mathrm{D}_{50}$ of $48.0 \mathrm{~Gy}$ (95\% CI 43.6-65.9) and normalized dose-response gradient $\left(\gamma_{50}\right)$ of 0.83 (95\% CI $\left.0.37-1.31\right)$. The dose for $10 \%$ and $20 \%$ probability of complication $\left(\mathrm{D}_{10}\right.$ and $\mathrm{D}_{20}$, respectively) was $18.4 \mathrm{~Gy}$ and $28.6 \mathrm{~Gy}$, respectively. Barney et al (24) reported the highest toxicity rate, which might have been related to their high percentage of patients who had undergone previous systemic therapy. Eight studies were included in the analysis relating grade $\geq 3$ general GI toxicity to the Rx dose $(10-12,21-23,25,26)$. The plot demonstrated a dose response with a statistically significant probit model fit (Fig. 3). The model fitting parameters were $\mathrm{D}_{50}$ of $87.1 \mathrm{~Gy}(95 \%$ CI 69.1-194.4) and $\gamma_{50}$ of 1.22 (95\% CI 0.78-1.68). The $\mathrm{D}_{10}$ and $\mathrm{D}_{20}$ values were $50.6 \mathrm{~Gy}$ and $63.1 \mathrm{~Gy}$, respectively. The large $\mathrm{D}_{50}$ value of $87.1 \mathrm{~Gy}$ can be attributed to the low rates of grade $\geq 3$ general GI toxicity or insufficient data.

\section{Special Situations}

Clinically, the toxicity profile of patients with metastatic disease can be very different from that of patients with primary tumors. Clinicians reported that patients with met-astatic liver disease tolerated radiation treatment more than did patients with primary liver tumors.

However, the data were insufficient to model each cohort separately; thus, it was decided to pool the data for this analysis. The types of tumors (metastatic and primary) in the various studies included in the analysis have been explicitly noted in the figures.

Because the liver is a parallel structure, it is amenable to reirradiation when new tumors subsequently occur in nearby areas of the liver. In cases of reirradiation, the dose-volume analysis accounting for anatomic changes should ideally be considered. The analysis for reirradiation cases should consider liver shrinkage and regeneration after SBRT using deformable image registration techniques. The University of Colorado group (36) reported a maximum total liver volume decrease of $\sim 20 \%$ (the result of liver shrinkage) at 3 to 6 months after SBRT, followed by a recovery and plateau to $\sim 10 \%$ less than the pretreatment volume at 12 months after SBRT. The likely reason for the liver volume not returning to the initial baseline value is the initial tumor-related expansion of the liver before treatment. The hypothesis from the University of Colorado group was that the tumor is likely to contribute 
additional volume to the liver in a combination of malignant cells, local tissue edema, and inflammation. Irradiation of the tumor seems to remove the sources of this artificially increased liver volume.

Although it would have been ideal to analyze additional dose-volume relationships (eg, absolute and relative liver volumes receiving certain doses and doses to nonhepatic GI structures), the work was limited by the reported metrics. Theoretically, the general GI toxicity should be a function of the dose delivered to the individual GI organs; however, in the absence of dosimetric data for each individual organ, we used the Rx doses. Seven studies (Table 2) reported some form of PTV dose as the Rx dose, including (1) 95\% or 97\% PTV coverage dose; or (2) an isodose line that should be capable of covering the PTV. Although these definitions of PTV dose were heterogeneous, they were sufficiently compatible. Given the absence of additional details in most of the reports, we directly used the Rx dose as a surrogate for the critical structure dose. BED conversions were not possible because most of the reports did not include the fractionation and GI toxicity outcomes per patient. Furthermore, the geometry of each tumor will differ in terms of the proximity to critical GI structures; thus, more detailed reporting of GI dose distributions and toxicities in future studies are needed to achieve more complete understanding. Although many inherent assumptions exist in using the Rx dose as a surrogate for specific GI organ doses, the model fits for GI toxicity proved significant (Figs. 2 and 3).

\section{Recommended Dose-Volume Objectives}

The Quantitative Analysis for Normal Tissue Effect in the Clinic (QUANTEC) liver report (19) has recommended 13 Gy (3 fractions) and 18 Gy (6 fractions) as MLD limits for primary disease and $15 \mathrm{~Gy}$ ( 3 fractions) and $20 \mathrm{~Gy}$ in (6 fractions) as MLD limits for metastatic lesions. Our toxicity analysis (Fig. 1) shows that the QUANTEC-recommended MLD limits would likely result in acceptable grade 3 liver enzyme toxicity risks $(<20 \%$ probability).

Although the dosimetric data were not sufficient to analyze the $700-\mathrm{cm}^{3}$ normal liver dose criteria, many investigators reported using $\geq 700 \mathrm{~cm}^{3}$ to $\geq 15$ Gy or $\geq 700 \mathrm{~cm}^{3}$ to $\geq 17$ Gy as treatment planning guidelines for patients receiving treatment in 3 to 6 fractions. Summing up the grade $\geq 3$ general GI toxicities from the studies that used the $\geq 700 \mathrm{~cm}^{3}$ to $\geq 15$ Gy or $\geq 700 \mathrm{~cm}^{3}$ to $\geq 17$ Gy planning guideline (excluding the single-fraction study by Goodman et al [10]), the toxicity rate was $9.3 \%$ (11 of 118 ) with $68 \%$ CIs of $6.6 \%$ to $12.8 \%$. Huang et al (22) was the only study that used the $\geq 700 \mathrm{~cm}^{3}$ to $\geq 15$ Gy planning constraint in HCC patients and reported grade $\geq 2$ GI toxicity. They reported a toxicity rate of $28 \%$ (10 of 36) (22). The HCC data from Son et al (20) suggested sparing $2800 \mathrm{~cm}^{3}$ to 318 Gy in 3 fractions.

As a third consideration, the analysis (Fig. 3) showed that a mean Rx dose of $50 \mathrm{~Gy}$ in 3 to 6 fractions would result in a grade $\geq 3$ general GI toxicity risk of $<10 \%$.

Thus, based on our analysis, we believe that the initial QUANTEC recommendations remain reasonable with respect to liver enzyme changes and general GI toxicity. We did not evaluate 
the decline in liver function as an endpoint; however, a recent study by Velec et al (29) suggested an MLD limit of $<18$ Gy (6 fractions) when considering the decline in liver function as an endpoint for patients with HCC. Further research is needed to validate any of the "guidelines" related to the decline in liver function as an endpoint for primary liver cancer.

\section{Future Studies}

In the present work, a number of factors limited our quantitative modeling and analysis, including the availability of detailed dose-volume histogram (DVH) data, mean, maximum, and minimum dose points of OARs, and the accurate conversion of physical doses to BEDs. Five of the studies included in Table 2 used the maximum dose of $15 \mathrm{~Gy}$ to $\geq 700 \mathrm{~cm}^{3}$ of normal liver as a treatment planning guideline. However, not all studies reported on the achieved doses and instead reported only the Rx doses or mean liver doses.

In future work, it would be beneficial to explicitly study the toxicity differences among patients with liver metastases, hepatocellular carcinoma, and intrahepatic cholangiocarcinoma. Dose-response analyses could be improved if investigators reported the achieved dose-volume metrics used for treatment planning guidance, including the full absolute DVHs (doses in Gy vs volume in $\mathrm{cm}^{3}$ ) for OARs. The minimum standards for reporting treatment outcomes in future studies outlined in the next section should include these factors and other relevant data to improve our understanding of the normal tissue response to liver SBRT. Currently, many journals allow for the inclusion of supplemental data that can be used to provide detailed DVH information and additional dosimetric details.

\section{Reporting Standards for Outcomes}

In the future, we suggest including the following parameters to enable readers to fully understand the reported data and to facilitate the pooling of data from multiple studies:

- Total liver volume, excluding the GTV

- Percentage of target volume covered by the Rx dose and the normalization isodose line

- A comprehensive set of dose metrics derived from the liver minus the GTV; this should include the mean liver doses, volume receiving $\geq 15 \mathrm{~Gy}$, liver $\mathrm{rV}_{\text {doses }}$ and doses to threshold volumes $\left(\mathrm{eg}, 800 \mathrm{~cm}^{3}\right)$.

- Detailed DVH data and the corresponding number of treatment fractions to allow for the accurate conversion of physical doses to BEDs.

- $\quad$ GTV to PTV margin and the method or methods used for target motion management and treatment localization, including the use and type of imageguided radiotherapy system

- $\quad$ Liver function, including the baseline and follow-up Child-Pugh scores, baseline albumin-bilirubin and follow-up scores, baseline liver enzymes, underlying liver disease (hepatitis B or C or any cirrhosis), baseline and follow-up platelet count, 
other conditions or therapies (eg, chemotherapy status) that might influence the treatment response, and cancer status (complete response, partial response, stable disease vs progressive disease)

- $\quad$ Previous systemic, regional, and local therapies

- $\quad$ Rates of toxicity using the CTCAE, version 4.0, grading scale or equivalent grading scale

- Explicit grade and cause of liver failure or, in the event that no liver failure toxicities occurred, that should be explicitly stated

- The certainty of the diagnosis of radiation-induced liver injury; in studies using endpoints that are not liver specific, a description of what was used to consider nonliver causes of toxicity would be helpful, including doses to adjacent upper abdominal structures, including bowel, stomach, and duodenum and volumes and doses to $0.5,5$, and $25 \mathrm{~cm}^{3}$

\section{Acknowledgments}

A.J. was supported in part by grant nos. 1RO1CA129182 and P30 CA008748 from the National Cancer Institute. E.Y. was supported in part by grant no. P30 CA008748 from the National Cancer Institute.

\section{References}

1. Ferlay J, Shin HR, Bray F, et al. Estimates of worldwide burden of cancer in 2008: Globocan 2008. Int J Cancer. 2010; 127:2893-2917. [PubMed: 21351269]

2. Paley MR, Ros PR. Hepatic metastases. Radiol Clin North Am. 1998; 36:349-363. [PubMed: 9520987]

3. Gayowski TJ, Iwatsuki S, Madariaga JR, et al. Experience in hepatic resection for metastatic colorectal cancer: Analysis of clinical and pathologic risk factors. Surgery. 1994; 116:703-711. [PubMed: 7940169]

4. Fong Y, Cohen AM, Fortner JG, et al. Liver resection for colorectal metastases. J Clin Oncol. 1997; 15:938-946. [PubMed: 9060531]

5. Aloia TA, Vauthey J-N, Loyer EM, et al. Solitary colorectal liver metastasis: Resection determines outcome. Arch Surg. 2006; 141:460-467. [PubMed: 16702517]

6. Herfarth KK, Debus J, Wannenmacher M. Stereotactic radiation therapy of liver metastases: Update of the initial phase I/II trial. Front Radiat Ther Oncol. 2004; 38:100-105. [PubMed: 15458194]

7. Wulf J, Hädinger U, Oppitz U, et al. Stereotactic radiotherapy of targets in the lung and liver. Strahlenther Onkol. 2001; 177:645-655. [PubMed: 11789403]

8. Schefter TE, Kavanagh BD, Timmerman RD, et al. A phase I trial of stereotactic body radiation therapy (SBRT) for liver metastases. Int J Radiat Oncol Biol Phys. 2005; 62:1371-1378. [PubMed: 16029795]

9. Rusthoven KE, Kavanagh BD, Cardenes H, et al. Multi-institutional phase I/II trial of stereotactic body radiation therapy for liver metastases. J Clin Oncol. 2009; 27:1572-1578. [PubMed: 19255321]

10. Goodman KA, Wiegner EA, Maturen KE, et al. Dose-escalation study of single-fraction stereotactic body radiotherapy for liver malignancies. Int J Radiat Oncol Biol Phys. 2010; 78:486493. [PubMed: 20350791]

11. Lee MT, Kim JJ, Dinniwell R, et al. Phase I study of individualized stereotactic body radiotherapy of liver metastases. J Clin Oncol. 2009; 27:1585-1591. [PubMed: 19255313] 
12. Bujold A, Massey CA, Kim JJ, et al. Sequential phase I and II trials of stereotactic body radiotherapy for locally advanced hepatocellular carcinoma. J Clin Oncol. 2013; 31:1631-1639. [PubMed: 23547075]

13. Tse RV, Hawkins M, Lockwood G, et al. Phase I study of individualized stereotactic body radiotherapy for hepatocellular carcinoma and intrahepatic cholangiocarcinoma. J Clin Oncol. 2008; 26:657-664. [PubMed: 18172187]

14. Andolino DL, Johnson CS, Maluccio M, et al. Stereotactic body radiotherapy for primary hepatocellular carcinoma. Int J Radiat Oncol Biol Phys. 2011; 81:e447-e453. [PubMed: 21645977]

15. Méndez Romero A, Wunderink W, Hussain SM, et al. Stereotactic body radiation therapy for primary and metastatic liver tumors: A single institution phase I-II study. Acta Oncol. 2006; 45:831-837. [PubMed: 16982547]

16. Kavanagh BD, Miften M, Rabinovitch RA. Advances in treatment techniques: Stereotactic body radiation therapy and the spread of hypofractionation. Cancer J. 2011; 17:177-181. [PubMed: 21610471]

17. McCammon R, Schefter TE, Gaspar LE, et al. Observation of a doseecontrol relationship for lung and liver tumors after stereotactic body radiation therapy. Int J Radiat Oncol Biol Phys. 2009; 73:112-118. [PubMed: 18786780]

18. Dawson LA, Normolle D, Balter JM, et al. Analysis of radiation-induced liver disease using the Lyman NTCP model. Int J Radiat Oncol Biol Phys. 2002; 53:810-821. [PubMed: 12095546]

19. Pan CC, Kavanagh BD, Dawson LA, et al. Radiation-associated liver injury. Int J Radiat Oncol Biol Phys. 2010; 76:S94-S100. [PubMed: 20171524]

20. Son SH, Choi BO, Ryu MR, et al. Stereotactic body radiotherapy for patients with unresectable primary hepatocellular carcinoma: Dose-volumetric parameters predicting the hepatic complication. Int J Radiat Oncol Biol Phys. 2010; 78:1073-1080. [PubMed: 20207492]

21. Kang JK, Kim MS, Cho CK, et al. Stereotactic body radiation therapy for inoperable hepatocellular carcinoma as a local salvage treatment after incomplete transarterial chemoembolization. Cancer. 2012; 118:5424-5431. [PubMed: 22570179]

22. Huang WY, Jen YM, Lee MS, et al. Stereotactic body radiation therapy in recurrent hepatocellular carcinoma. Int J Radiat Oncol Biol Phys. 2012; 84:355-361. [PubMed: 22342300]

23. Bae SH, Kim MS, Cho CK, et al. Feasibility and efficacy of stereotactic ablative radiotherapy for Barcelona Clinic liver cancer-C stage hepatocellular carcinoma. J Korean Med Sci. 2013; 28:213219. [PubMed: 23400333]

24. Barney BM, Olivier KR, Miller RC, et al. Clinical outcomes and toxicity using stereotactic body radiotherapy (SBRT) for advanced cholangiocarcinoma. Radiat Oncol. 2012; 7:67. [PubMed: 22553982]

25. Vautravers-Dewas C, Dewas S, Bonodeau F, et al. Image-guided robotic stereotactic body radiation therapy for liver metastases: Is there a dose response relationship? Int J Radiat Oncol Biol Phys. 2011; 81:e39-e47. [PubMed: 21377292]

26. Barney BM, Markovic SN, Laack NN, et al. Increased bowel toxicity in patients treated with a vascular endothelial growth factor inhibitor (VEGFI) after stereotactic body radiation therapy (SBRT). Int J Radiat Oncol Biol Phys. 2013; 87:73-80. [PubMed: 23920388]

27. Jabbour SK, Hashem SA, Bosch W, et al. Upper abdominal normal organ contouring guidelines and atlas: A radiation therapy oncology group consensus. Pract Radiat Oncol. 2014; 4:82-89. [PubMed: 24890348]

28. Ben-Josef E, Normolle D, Ensminger WD, et al. Phase II trial of high-dose conformal radiation therapy with concurrent hepatic artery floxuridine for unresectable intrahepatic malignancies. $\mathrm{J}$ Clin Oncol. 2005; 23:8739-8747. [PubMed: 16314634]

29. Velec M, Haddad CR, Craig T, et al. Predictors of liver toxicity following stereotactic body radiation therapy for hepatocellular carcinoma. Int J Radiat Oncol Biol Phys. 2017; 97:939-946. [PubMed: 28333016]

30. Lasley FD, Mannina EM, Johnson CS, et al. Treatment variables related to liver toxicity in patients with hepatocellular carcinoma, Child-Pugh class A and B enrolled in a phase 1-2 trial of stereotactic body radiation therapy. Pract Radiat Oncol. 2015; 5:e443-e449. [PubMed: 25899219] 
31. Toesca DAS, Osmundson EC, von Eyben R, et al. Assessment of hepatic function decline after stereotactic body radiation therapy for primary liver cancer. Pract Radiat Oncol. 2017; 7:173-182. [PubMed: 28343896]

32. Liang SX, Zhu XD, Xu ZY, et al. Radiation-induced liver disease in three-dimensional conformal radiation therapy for primary liver carcinoma: The risk factors and hepatic radiation tolerance. Int J Radiat Oncol Biol Phys. 2006; 65:426-434. [PubMed: 16690430]

33. Burman C, Kutcher GJ, Emami B, et al. Three-dimensional photon treatment planning report of the collaborative working group on the evaluation of treatment planning for external photon beam radio-therapy: Fitting of normal tissue tolerance data to an analytic function. Int J Radiat Oncol Biol Phys. 1991; 21:123-135. [PubMed: 2032883]

34. Källman P, Ågren A, Brahme A. Tumour and normal tissue responses to fractionated non-uniform dose delivery. Int J Radiat Biol. 1992; 62:249-262. [PubMed: 1355519]

35. Morgan, BJ. Analysis of quantal response data. In: Smith, RL, Tong, H, Keiding, N. , et al., editors. Monographs on Statistics and Applied Probability. Boca Raton, FL: CRC Press; 1992. 46

36. Stinauer MA, Diot Q, Westerly DC, et al. Fluorodeoxyglucose positron emission tomography response and normal tissue regeneration after stereotactic body radiotherapy to liver metastases. Int J Radiat Oncol Biol Phys. 2012; 83:e613-e618. [PubMed: 22494588] 


\section{Summary}

A quantitative review of published data on radiation-induced liver and gastrointestinal (GI) normal tissue toxicities in liver stereotactic body radiation therapy (SBRT) is performed. The report presents normal tissue dose-response parameters, recommendations for dose-volume limits for liver SBRT, and outlines standards for future reporting of liver and GI dosimetric and clinical data. 


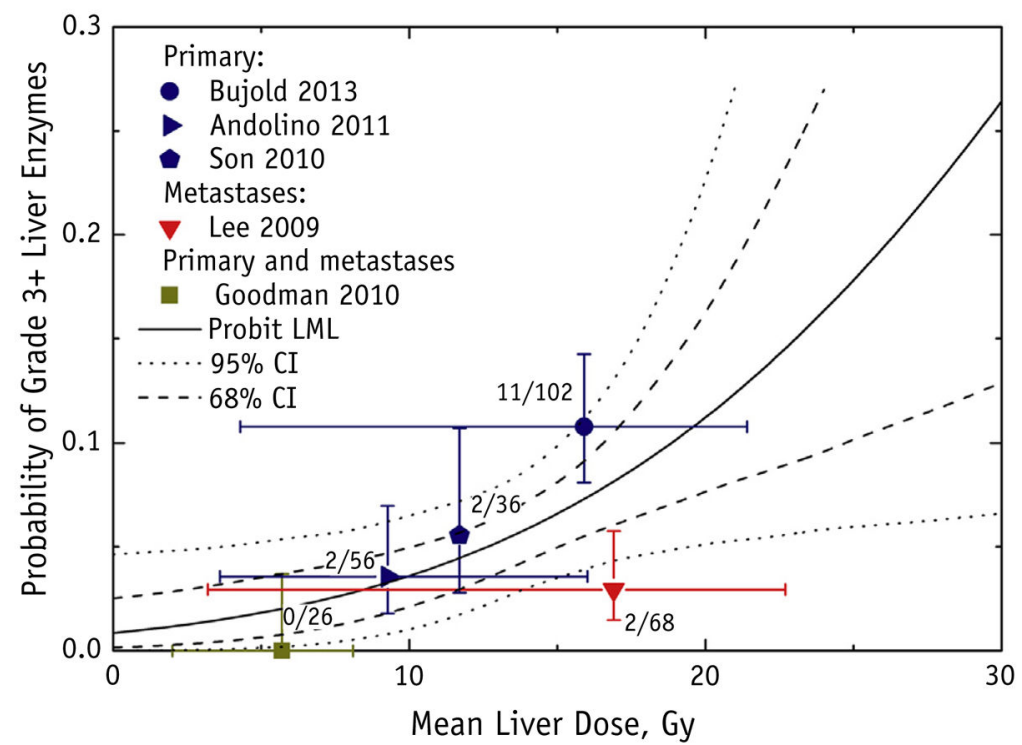

Fig. 1.

Grade $\geq 3$ liver enzyme toxicity as a function of mean liver dose (MLD), with probit model fit and $95 \%$ and 68\% confidence intervals (CIs). Dose "error bars" (horizontal axis) for each data point represent range of reported doses for each study; toxicity "error bars" (vertical axis) represent binomial $68 \%$ CIs. The number of patients who developed toxicity of the total number of patients for each study is displayed next to the data point. All studies excluded the gross tumor volume in the MLD calculation, with the exception of Son et al (20). Probit model fitting failed to establish the upper confidence limit for the dose to $50 \%$ of the target, and the null hypothesis of no dose response was not rejected $(P=.10)$; therefore, we could not exclude that the incidence of liver enzyme complications was independent of the dose; the probit model fit is displayed for reference. Abbreviation: LML $=\log$ maximum likelihood. 


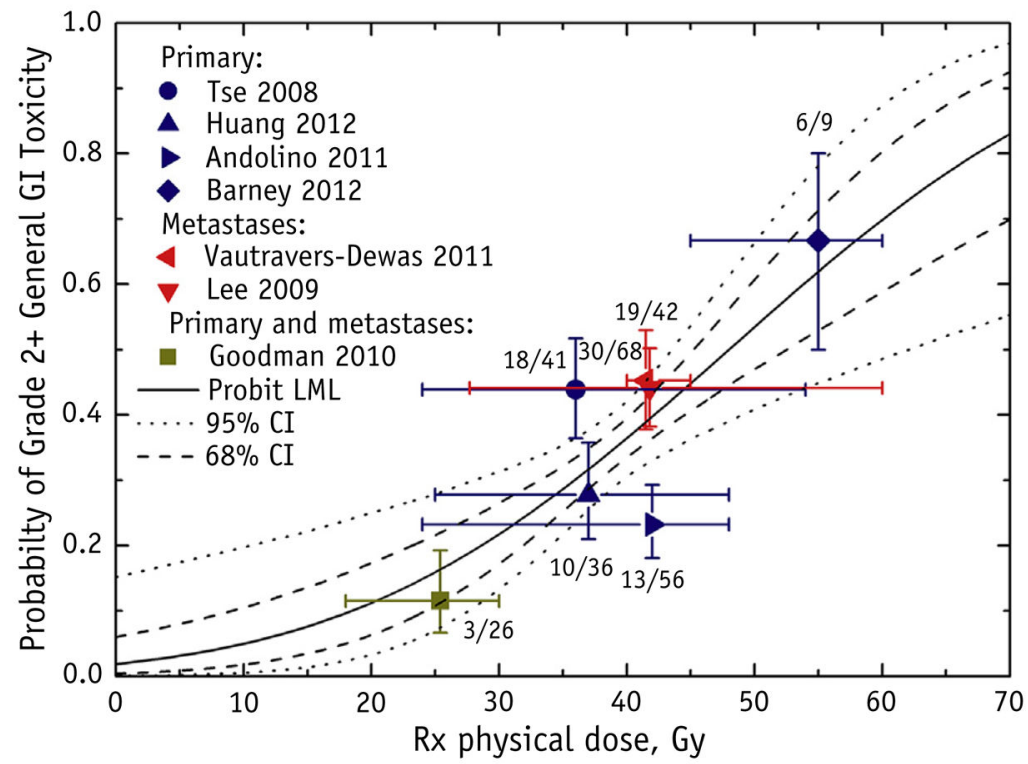

Fig. 2.

Grade $\geq 2$ general gastrointestinal (GI) toxicity as a function of the prescription (Rx) physical dose to the target, with the probit model result (maximum likelihood parameter fitting) and $95 \%$ and $68 \%$ confidence intervals (CIs). General GI toxicities were defined as fatigue, nausea, diarrhea, gastritis, ulcers, GI area pain, and colitis. The target Rx dose definition is provided in Table 2. Each data point was placed at the reported mean or median dose and reported complication rate; horizontal error bars represent the reported ranges, and the vertical error bars represent binomial $68 \%$ CIs. The number of patients who developed toxicity of the total number of patients for each study is displayed next to the data point. The study by Andolino et al (14) did not distinguish between grade 1 and 2 general GI toxicities. Abbreviation: $\mathrm{LML}=\log$ maximum likelihood. 


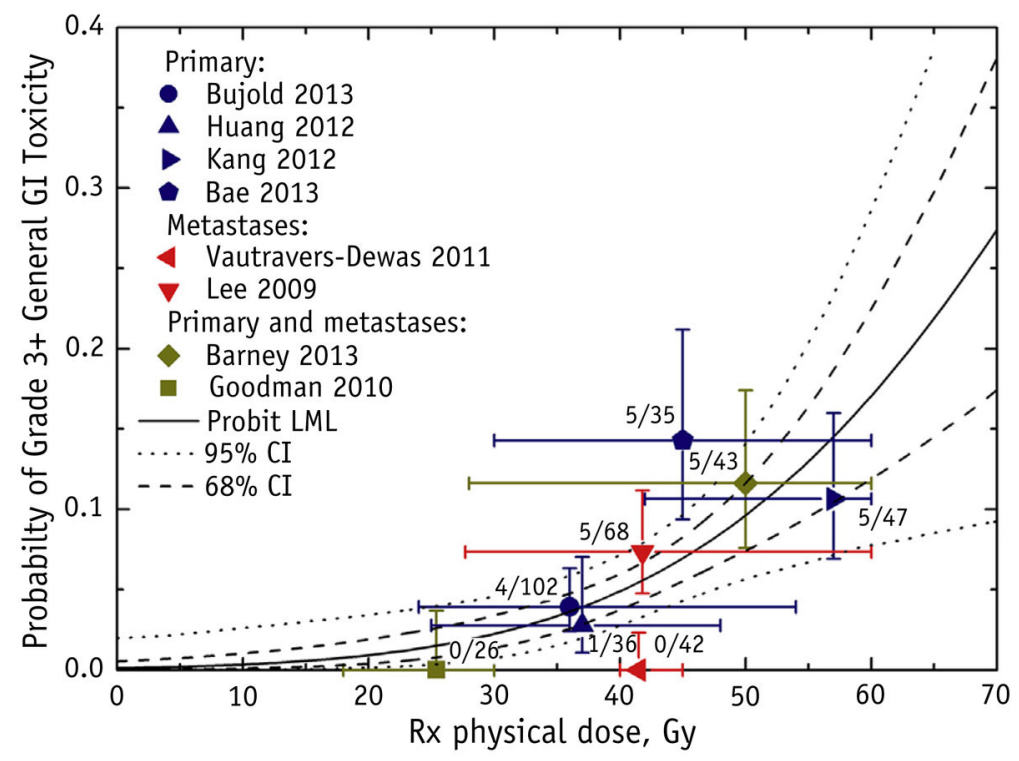

Fig. 3.

Grade $\geq 3$ general gastrointestinal (GI) toxicity as a function of the prescription (Rx) physical dose to the target, with the probit model result (maximum likelihood parameter fitting) and $95 \%$ and $68 \%$ confidence intervals (CIs). General GI toxicities were defined as fatigue, nausea, diarrhea, gastritis, ulcers, GI area pain, and colitis. The target RX dose definition is provided in Table 2. Each data point is placed at the reported mean or median dose and reported complication rate; the horizontal error bars represent the reported ranges, and the vertical error bars represent binomial $68 \%$ CIs. The number of patients who developed toxicity out of the total number of patients for each study is displayed next to the data point. Abbreviation: $\mathrm{LML}=\log$ maximum likelihood. 


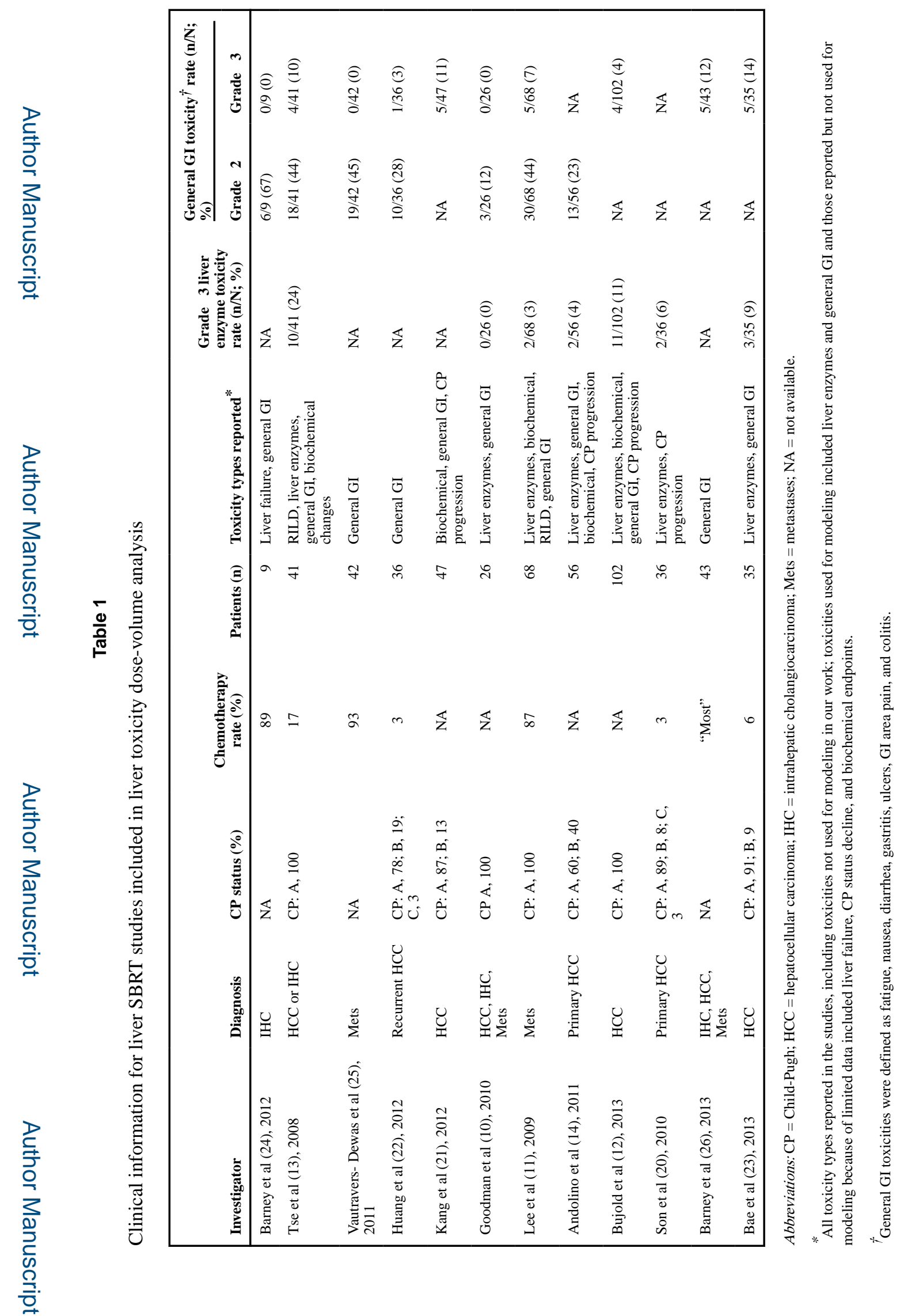

Int J Radiat Oncol Biol Phys. Author manuscript; available in PMC 2022 May 01. 


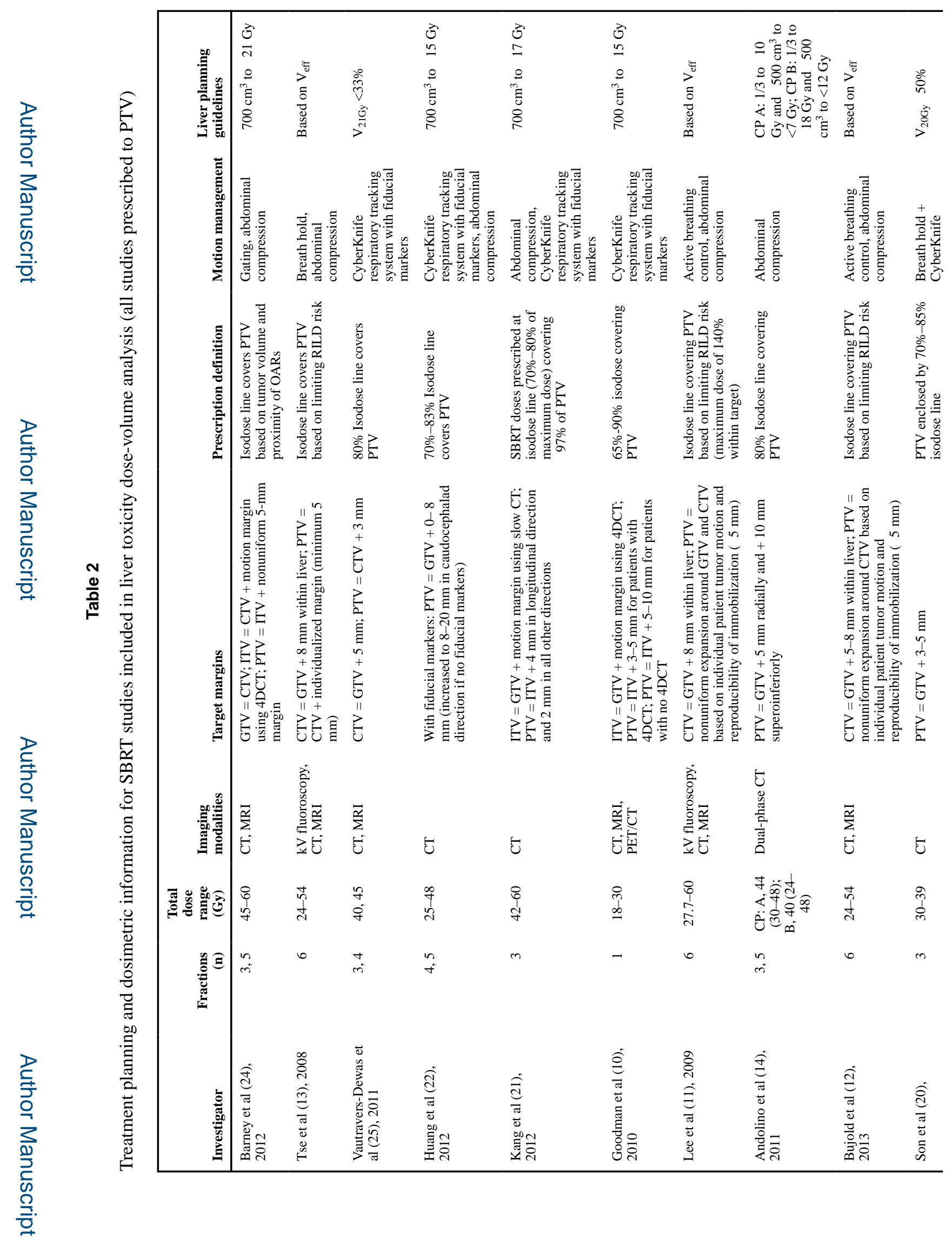

Int J Radiat Oncol Biol Phys. Author manuscript; available in PMC 2022 May 01. 


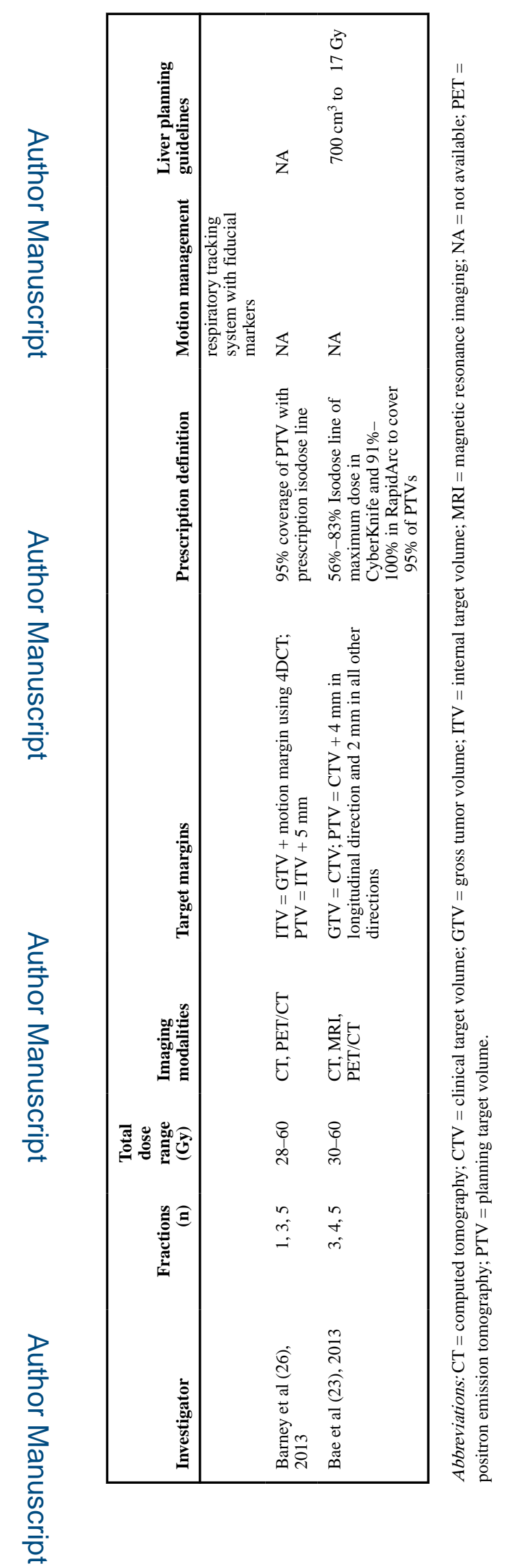

Int J Radiat Oncol Biol Phys. Author manuscript; available in PMC 2022 May 01. 
Table 3

Summary of dose-response and probit model fit parameters for considered endpoints

\begin{tabular}{|llll|}
\hline Endpoint & Events (n)/patients $(\mathbf{n})$ & $\mathbf{D}_{\mathbf{5 0}}(\mathbf{G y})$ & $\boldsymbol{\gamma}_{\mathbf{5 0}}{ }^{*}$ \\
\hline $\begin{array}{l}\text { Grade } \\
\quad 17 / 288\end{array}$ & $40.8(25.5-\infty)$ & $0.95(0.58-1.44)$ \\
$\quad 3$ liver enzymes & & & \\
$\begin{array}{l}\text { Grade } \\
\quad 2 \mathrm{GI}\end{array}$ & $99 / 278$ & $48.0(43.6-65.9)$ & $0.83(0.37-1.31)$ \\
$\begin{array}{l}\text { Grade } \\
\text { 23 GI }\end{array}$ & $25 / 399$ & $87.1(69.1-194.4)$ & $1.22(0.78-1.68)$ \\
\hline
\end{tabular}

Abbreviations: $\gamma_{50}=$ normalized dose-response gradient; $\mathrm{D}_{50}=$ dose for $50 \%$ complication probability.

Data in parentheses are $95 \%$ confidence intervals.

${ }^{*} \gamma 50=1 /[\mathrm{m} \sqrt{ }(2 \pi)]$, where $\mathrm{m}$ is a slope factor. 